\title{
Accurate Rotational Rest Frequencies for Ammonium Ion Isotopologues
}

\author{
José L. Doménech ${ }^{1}$ (D), Stephan Schlemmer ${ }^{2}$ (i), and Oskar Asvany ${ }^{2}$ (I) \\ ${ }^{1}$ Instituto de Estructura de la Materia (IEM-CSIC), Serrano 123, E-28006 Madrid, Spain \\ ${ }^{2}$ I. Physikalisches Institut, Universität zu Köln, Zülpicher Str. 77, D-50937 Köln, Germany; asvany@ph1.uni-koeln.de \\ Received 2018 August 16; revised 2018 August 31; accepted 2018 September 4; published 2018 October 24
}

\begin{abstract}
We report rest frequencies for rotational transitions of the deuterated ammonium isotopologues $\mathrm{NH}_{3} \mathrm{D}^{+}, \mathrm{NH}_{2} \mathrm{D}_{2}^{+}$, and $\mathrm{NHD}_{3}^{+}$, measured in a cryogenic ion trap machine. For the symmetric tops $\mathrm{NH}_{3} \mathrm{D}^{+}$and $\mathrm{NHD}_{3}^{+}$, one and three transitions are detected, respectively, and five transitions are detected for the asymmetric top $\mathrm{NH}_{2} \mathrm{D}_{2}^{+}$. $\mathrm{While}$ the lowest frequency transition of $\mathrm{NH}_{3} \mathrm{D}^{+}$was already known in the laboratory and space, this work enables the future radio astronomical detection of the two other isotopologues.
\end{abstract}

Key words: ISM: molecules - methods: laboratory: molecular - molecular data - techniques: spectroscopic

\section{Introduction}

Nitrogen is one of the most abundant elements in the local universe, and has a notably rich chemistry, with more than 70 nitrogen-containing molecules identified in space to date. ${ }^{3}$ Two of the most abundant nitrogen-bearing molecules in the interstellar medium (ISM) are $\mathrm{N}_{2}$ and $\mathrm{NH}_{3}$, which are predicted to be present in many different media, from cold dark clouds (see, e.g., Nejad et al. 1990) or protostellar cores (see, e.g., Aikawa et al. 2008) to active galactic nuclei (see, e.g., Harada et al. 2010). $\mathrm{N}_{2}$, being a homonuclear diatomic molecule, cannot be observed through its (electric-dipole) rotation or vibration transitions. It can be observed, however, through electronic transitions in the far-ultraviolet, with only one direct observation reported by Knauth et al. (2004), or by its frequently used proxy, the diazenylium ion $\left(\mathrm{N}_{2} \mathrm{H}^{+}\right)$. Ammonia, in turn, is the first polyatomic molecule identified in space (Cheung et al. 1968), and since then has been observed in many environments. To date, all deuterated isotopologues of ammonia, $\mathrm{NH}_{2} \mathrm{D}, \mathrm{NHD}_{2}$, and $\mathrm{ND}_{3}$, have been observed (see e.g., Harju et al. 2017 for a recent account), as well as ${ }^{15} \mathrm{NH}_{3}$ and ${ }^{15} \mathrm{NH}_{2} \mathrm{D}$ (Gerin et al. 2009).

The astrophysical relevance of the ammonium ion, $\mathrm{NH}_{4}^{+}$, stems from its role as a gas-phase forebear of the ammonia molecule through its dissociative recombination. The full family of nitrogen hydrides is initiated by the reaction $\mathrm{N}^{+}+\mathrm{H}_{2} \rightarrow \mathrm{NH}^{+}+\mathrm{H}$ (Zymak et al. 2013). Subsequent exothermic $\mathrm{H}_{2}$ abstraction reactions lead to the formation of $\mathrm{NH}_{2}^{+}, \mathrm{NH}_{3}^{+}$, and $\mathrm{NH}_{4}^{+}$, and their recombination with electrons form the neutral hydrides $\mathrm{NH}, \mathrm{NH}_{2}$, and $\mathrm{NH}_{3}$. Ammonia is supposed to be mainly depleted onto grains at the temperatures of cold dark clouds $(T \sim 10 \mathrm{~K})$. On the other hand, the grains can free $\mathrm{NH}_{3}$ molecules upon heating by shocks or in irradiated regions, increasing its gas-phase concentration and leading to appreciable quantities of $\mathrm{NH}_{4}^{+}$, formed by the proton transfer from $\mathrm{H}_{3}^{+}$to $\mathrm{NH}_{3}$. Actually, $\mathrm{NH}_{3}$ has one of the highest proton affinities (PA) of simple interstellar molecules $\left(\mathrm{PA}\left[\mathrm{NH}_{3}\right]=\right.$ $8.85 \mathrm{eV}$ ), including $\mathrm{H}_{2}$ (which has $\mathrm{PA}\left[\mathrm{H}_{2}\right]=4.39 \mathrm{eV}$ ), so $\mathrm{NH}_{4}^{+}$, once formed, remains stable against further collisions with $\mathrm{H}_{2}$.

$\mathrm{NH}_{4}^{+}$has tetrahedral symmetry without a permanent electricdipole moment. Therefore, it is untraceable by radio astronomy

\footnotetext{
3 CDMS 2018, https://www.astro.uni-koeln.de/cdms/molecules.
}

through its rotational transitions. On the other hand, the deuterated isotopologues $\mathrm{NH}_{3} \mathrm{D}^{+}, \mathrm{NH}_{2} \mathrm{D}_{2}^{+}$, and $\mathrm{NHD}_{3}^{+}$have sizable permanent electric-dipole moments (approximately $0.26 \mathrm{D}, 0.29 \mathrm{D}$, and $0.24 \mathrm{D}$, respectively) due to the separation between the center of charge (which remains centered on the central $\mathrm{N}$ atom) and the center of mass (which is displaced towards the D atoms), thus making their detection feasible. Indeed, the detection of ammonium in space was claimed through the assignment of an emission line centered at $262,817 \mathrm{GHz}$ (observed both in Orion IRc2 and in Barnard $\mathrm{B} 1-\mathrm{bS}$ ) to the $1_{0}-0_{0}$ transition of $\mathrm{NH}_{3} \mathrm{D}^{+}$(Cernicharo et al. 2013). The laboratory rest frequency was derived from the analysis of the high-resolution infrared (IR) spectrum of the $\nu_{4}$ band, originally made by Nakanaga \& Amano (1986) and considerably improved by Doménech et al. (2013). The rest frequency was later confirmed, and its uncertainty was substantially decreased, by the direct measurement of the rotational transition by Stoffels et al. (2016).

Besides their importance as tracers of $\mathrm{NH}_{4}^{+}$, the deuterated isotopologues are also relevant regarding the subject of deuterium fractionation. In cold dark clouds, when $\mathrm{CO}$ freezes onto grains and its abundance falls below that of $\mathrm{HD}$, deuteration of other species becomes efficient. This can be explained by the exothermicity $(\sim 230 \mathrm{~K})$ of the reaction $\mathrm{H}_{3}^{+}+\mathrm{HD} \rightarrow \mathrm{H}_{2} \mathrm{D}^{+}+\mathrm{H}_{2}$ and the subsequent deuteron transfer to other molecules. Similarly, the exothermic reactions of $\mathrm{H}_{2} \mathrm{D}^{+}$and $\mathrm{HD}_{2}^{+}$with $\mathrm{HD}$ efficiently produce $\mathrm{HD}_{2}^{+}$and $\mathrm{D}_{3}^{+}$(Hugo et al. 2009) in case of extreme depletion, and the subsequent deuteron transfer to ammonia leads to the deuterated forms of $\mathrm{NH}_{4}^{+}$e.g., $\mathrm{NH}_{3}+\mathrm{H}_{2} \mathrm{D}^{+} \rightarrow$ $\mathrm{NH}_{3} \mathrm{D}^{+}+\mathrm{H}_{2}$. Furthermore, the zero-point energy differences of other reactions involving $\mathrm{H}$ or $\mathrm{D}$ atoms at temperatures of cold dark clouds ( $T \sim 10 \mathrm{~K}$ ) also enhance deuteration (Millar 2003). For the case of nitrogen, the reaction $\mathrm{N}^{+}+\mathrm{HD} \rightarrow \mathrm{ND}^{+}+\mathrm{H}$ has a lower endothermicity than the corresponding reaction with $\mathrm{H}_{2}$ mentioned above (Grozdanov et al. 2016), leading to an enrichment in deuterated ammonium and ammonia isotopologues. The detected abundances of deuterated variants of ammonia are orders of magnitude higher than what is expected based on the $[\mathrm{D}] /[\mathrm{H}]$ abundance ratio $\left(2.35 \times 10^{-5}\right.$ in the local universe; Linsky et al. 2006). Detection of other deuterated ammonium isotopologues besides $\mathrm{NH}_{3} \mathrm{D}^{+}$will clearly help to constrain and to understand the conditions of formation and distribution of ammonia molecules and, possibly, other nitrogen-containing prebiotic species. 
The only previous spectroscopic laboratory data on the doubly and triply deuterated species $\left(\mathrm{NH}_{2} \mathrm{D}_{2}^{+}\right.$and $\left.\mathrm{NHD}_{3}^{+}\right)$that we are aware of are the recent publication of the analysis of the $\nu_{1}$ and $\nu_{6}$ infrared bands of $\mathrm{NH}_{2} \mathrm{D}_{2}^{+}$(Chang \& Nesbitt 2018) and the communication of preliminary results on the analysis of the $\nu_{1}$ band of $\mathrm{NHD}_{3}^{+}$(Chang \& Nesbitt 2013), both recorded at high resolution in a supersonic slit-jet discharge with an infrared difference-frequency laser spectrometer. In this work, we present direct and accurate laboratory measurements of the lowest frequency rotational transitions of $\mathrm{NH}_{3} \mathrm{D}^{+}, \mathrm{NH}_{2} \mathrm{D}_{2}^{+}$, and $\mathrm{NHD}_{3}^{+}$. The fundamental rotational frequency for $\mathrm{NH}_{3} \mathrm{D}^{+}$has already been published previously, and we only confirm its value, while our reported values for the other two isotopologues represent the first available sub-mm wave data.

\section{Experimental Methods}

The rotational transitions of the deuterated ammonium isotopologues have been measured in the Köln laboratories, exploiting the rotational state dependence of the attachment of He atoms to cations (Brünken et al. 2014, 2017; Doménech et al. 2017, 2018; Jusko et al. 2017). The experiment was performed in the $4 \mathrm{~K}$ trapping machine COLTRAP described by Asvany et al. (2010, 2014). The ions were generated in a storage ion source by bombarding the precursor gas mixture with electrons (energy 30-40 eV). For generating $\mathrm{NH}_{3} \mathrm{D}^{+}$and $\mathrm{NH}_{2} \mathrm{D}_{2}^{+}, \mathrm{NH}_{3}$ (Messer, $99.8 \%$ purity) and a 1:5 mixture of $\mathrm{D}_{2}$ (Linde, 99.8\%) and He (Linde, 99.999\%) were admitted to the ion source via two separate leakage valves. The approximate proportions were 1:1:5 for $\mathrm{NH}_{3} \mathrm{D}^{+}$and 1:2:10 for $\mathrm{NH}_{2} \mathrm{D}_{2}^{+}$. For $\mathrm{NHD}_{3}^{+}$, we applied a similar mixture using $\mathrm{ND}_{3}$ (Campro Scientific, 99\% atom D) and $\mathrm{H}_{2}$ (Linde, 99.9999\%). A pulse of several 10,000 mass-selected parent ions was injected into the $4 \mathrm{~K}$ cold 22-pole ion trap filled with about $10^{14} \mathrm{~cm}^{-3} \mathrm{He}$. During the trapping time of $750 \mathrm{~ms}$, cation-helium complexes were formed by three-body collisions. The detection of the resonant absorption of the admitted continuous wave (sub) millimeter radiation by the trapped cold parent cations was achieved by observing the decrease of the number of cationhelium complexes formed. For example, for recording a rotational transition of $\mathrm{NH}_{2} \mathrm{D}_{2}^{+}$, the $\mathrm{NH}_{2} \mathrm{D}_{2}^{+}-\mathrm{He}$ complexes are counted (typical counts are on the order of 2000) as a function of the (sub)millimeter-wave frequency. The (sub) millimeter-wave radiation was supplied by a rubidium atomic clock-referenced synthesizer (Rohde \& Schwarz SMF100A) driving a multiplier chain source (Virginia Diodes, Inc.), covering the range of $80-1100 \mathrm{GHz}$.

\section{Results}

We started our measurements by optimizing the experimental conditions using the known $1_{0}-0_{0}$ line of $\mathrm{NH}_{3} \mathrm{D}^{+}$, which has been measured by Stoffels et al. (2016) with the same ion trap technique. We confirm their value for $1_{0}-0_{0}$, as well as the inability of the applied setups to detect the $2_{0}-1_{0}$ and $2_{1}-1_{1}$ transitions. All experimental values of this work are summarized in Table 1.

For $\mathrm{NH}_{2} \mathrm{D}_{2}^{+}$, we based our search on the predictions from the high-resolution infrared vibration-rotation spectra of the $\nu_{1}$ and $\nu_{6}$ bands recently published by Chang \& Nesbitt (2018). In total, we searched for eight rotational lines of $\mathrm{NH}_{2} \mathrm{D}_{2}^{+}$of which five were detected. All measured and predicted transitions are included in Table 1. Figure 1 shows two example
Table 1

Frequencies of Pure Rotational Transitions (in $\mathrm{MHz}$ ) of Deuterated Ammonium Isotopologues

\begin{tabular}{|c|c|c|c|}
\hline $\mathrm{NH}_{3} \mathrm{D}^{+}$ & $J_{K}^{\prime} \leftarrow J_{K}^{\prime \prime}$ & This Work & Former Work $^{\mathrm{a}}$ \\
\hline & $1_{0} \leftarrow 0_{0}$ & $262816.8864(8)$ & $262816.904(15)$ \\
\hline \multirow[t]{9}{*}{$\mathrm{NH}_{2} \mathrm{D}_{2}^{+}$} & $J_{K_{a}^{\prime} K_{c}^{\prime}}^{\prime} \leftarrow J_{K_{a}^{\prime \prime} K_{c}^{\prime \prime}}^{\prime \prime}$ & This Work & Former Prediction \\
\hline & $1_{10} \leftarrow 1_{01}$ & $42273.4715(47)^{\mathrm{c}}$ & $42270(6)$ \\
\hline & $1_{11} \leftarrow 0_{00}$ & $248918.2760(8)$ & $248924(6)$ \\
\hline & $2_{02} \leftarrow 1_{11}$ & $412755.5374(27)$ & \\
\hline & $2_{12} \leftarrow 1_{01}$ & $455534.5852(16)$ & $455540(12)$ \\
\hline & $2_{21} \leftarrow 1_{10}$ & $540046.0112(75)$ & \\
\hline & $2_{20} \leftarrow 1_{11}$ & $560809.9582(37)$ & \\
\hline & $3_{03} \leftarrow 2_{12}$ & $632874.2(17)^{\mathrm{c}}$ & \\
\hline & $3_{13} \leftarrow 2_{02}$ & $656593.10(82)^{\mathrm{c}}$ & \\
\hline \multirow[t]{4}{*}{$\mathrm{NHD}_{3}^{+}$} & $J_{K}^{\prime} \leftarrow J_{K}^{\prime \prime}$ & This Work & \\
\hline & $1_{0} \leftarrow 0_{0}$ & $222228.9432(7)$ & \\
\hline & $2_{0} \leftarrow 1_{0}$ & $444415.7929(42)$ & \\
\hline & $2_{1} \leftarrow 1_{1}$ & $444421.2980(18)$ & \\
\hline
\end{tabular}

Notes. The final error is given in parentheses in units of the last digit

${ }^{\text {a }}$ Stoffels et al. (2016).

${ }^{\mathrm{b}}$ Chang \& Nesbitt (2018).

${ }^{\mathrm{c}}$ Our prediction.

measurements for $\mathrm{NH}_{2} \mathrm{D}_{2}^{+}$. All lines have been measured at least 7 times and fitted to Gaussian functions, from which line centers and widths were determined. The values quoted in Table 1 represent the combined mean and standard deviation of all measurements. During the measurements, care was taken to avoid power broadening. By this, the line widths are determined only by the Doppler broadening due to the kinetic temperature of the ions in the trap (nominal temperature of $T=4 \mathrm{~K}$ ) and also by a small contribution from the unresolved hyperfine splitting due to the quadrupole moments of the ${ }^{14} \mathrm{~N}$ and ${ }^{2} \mathrm{H}$ nuclei, both with spins of $I=1$. Effectively, we measure line widths corresponding to $T=10-20 \mathrm{~K}$ for $\mathrm{NH}_{2} \mathrm{D}_{2}^{+}$, depending on the observed line. The $1_{0}-0_{0}$ line of $\mathrm{NH}_{3} \mathrm{D}^{+}$seems to be more affected by hyperfine splitting, with an effective temperature of $T \approx 30 \mathrm{~K}$.

For $\mathrm{NHD}_{3}^{+}$, our search was guided by mass-scaling the rotational constants from the other isotopologues, as well as by an estimation based on the available IR-derived rotational constants from the Nesbitt group (Chang \& Nesbitt 2013). In contrast to $\mathrm{NH}_{3} \mathrm{D}^{+}$, we were able to measure not only the fundamental $1_{0}-0_{0}$ for $\mathrm{NHD}_{3}^{+}$, but also detected the $2_{0}-1_{0}$ and $2{ }_{1}-1_{1}$ transitions. The reason is a more advantageous partition function for $\mathrm{NHD}_{3}^{+}$, as well as more available millimeter-wave power at the lower transition frequencies of the heavier $\mathrm{NHD}_{3}^{+}$.

\section{Spectroscopic Parameters}

As accurate spectroscopic parameters and predictions for the ground state of $\mathrm{NH}_{3} \mathrm{D}^{+}$were already given by Stoffels et al. (2016) and Doménech et al. (2013), we treat here only the isotopologues $\mathrm{NH}_{2} \mathrm{D}_{2}^{+}$and $\mathrm{NHD}_{3}^{+}$. For $\mathrm{NH}_{2} \mathrm{D}_{2}^{+}$, the measured frequencies of the pure rotational lines collected in Table 1 were fit together with the ground state combination differences derived from Chang \& Nesbitt (2018) (two duplicated entries had to be trimmed) using the program PGOPHER (Western 2017), rendering the final set of parameters for the ground state given in Table 2 . Both sets of data were weighted according 


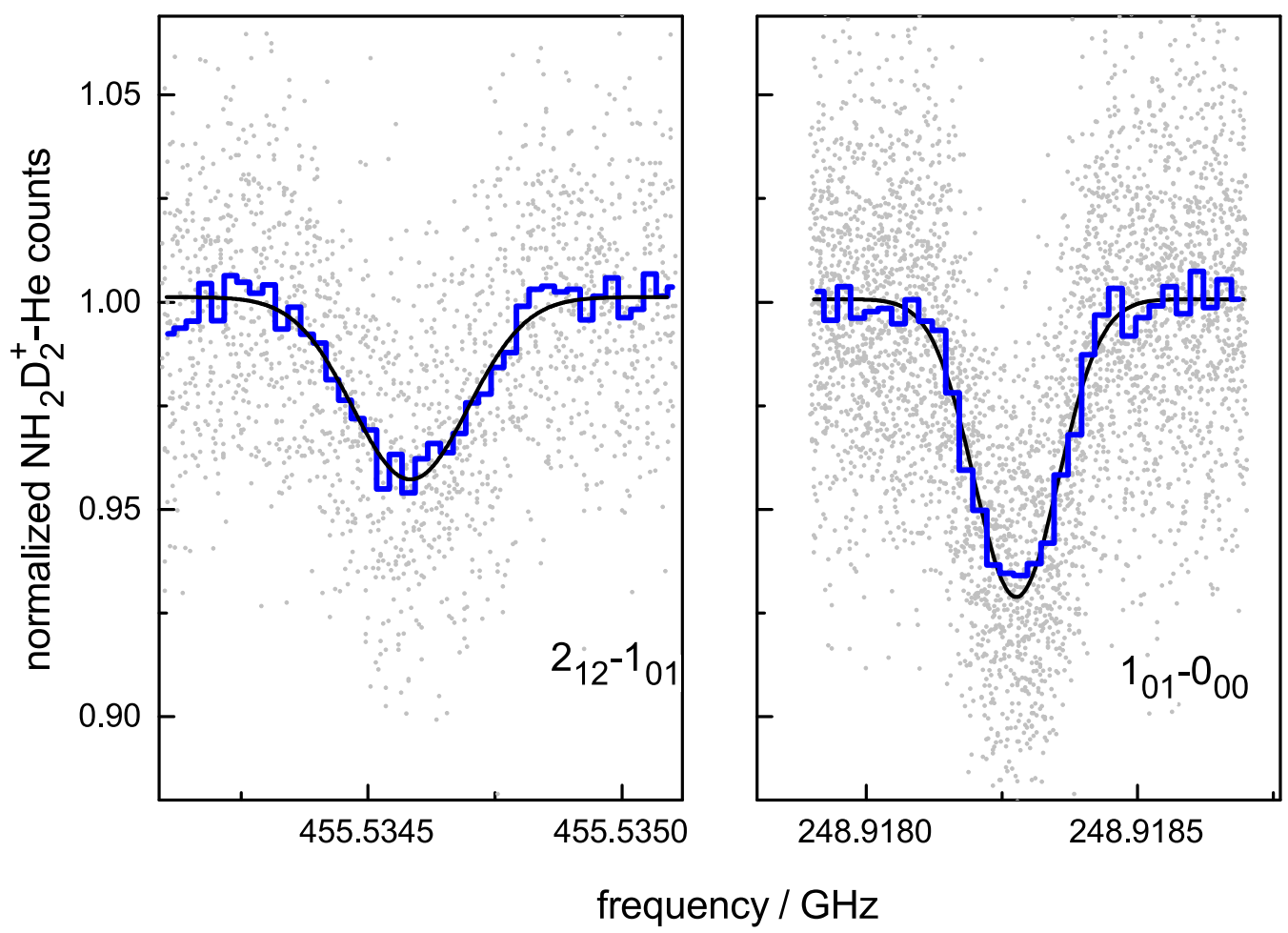

Figure 1. Example measurements of the $2_{12}-1_{01}$ and $1_{01}-0_{00}$ rotational transitions of $\mathrm{NH}_{2} \mathrm{D}_{2}^{+}$, recorded as a depletion signal of the normalized $\mathrm{NH}_{2} \mathrm{D}_{2}^{+}-\mathrm{He}$ counts. Gray dots are single measurements, the blue line is the average binned in $25 \mathrm{kHz}$ steps, and the black trace is a Gaussian fit.

Table 2

Derived Spectroscopic Parameters (in $\mathrm{MHz}$ ) from a Fit to All Measured Transitions

\begin{tabular}{clcc}
\hline \hline $\mathrm{NH}_{2} \mathrm{D}_{2}^{+}$ & Parameter & This Work & Former Work $^{\mathrm{a}}$ \\
\hline & $A$ & $145600.69(33)$ & $145601.7(12)$ \\
& $B$ & $118963.31(77)$ & $118966.3(12)$ \\
$C$ & $103324.30(63)$ & $103328.6(18)$ \\
& $\Delta_{J}$ & $1.698(46)$ & $2.10(9)$ \\
$\Delta_{J K}$ & $-0.85(24)$ & $-1.9(4)$ \\
& $\Delta_{K}$ & $3.08(24)$ & $3.87(3)$ \\
& $\delta_{J}$ & $0.371(15)$ & $0.18(6)$ \\
& $\delta_{K}$ & $0.01(20)$ & $0.9(3)$ \\
\hline $\mathrm{NHD}_{3}^{+}$ & Parameter & This Work & Former Work \\
\hline & $B$ & $111117.9794(6)$ & $111120.9(8)$ \\
& $D_{J}$ & $1.7539(2)$ & $2.01(7)$ \\
& $D_{J K}$ & $-1.3763(11)$ & $-2.0(2)$ \\
\hline
\end{tabular}

Notes. Numbers in parentheses are one standard deviation in units of the last digit.

${ }^{\mathrm{a}}$ Chang \& Nesbitt (2018).

${ }^{\mathrm{b}}$ Chang \& Nesbitt (2013).

to their different uncertainties, in the $\mathrm{kHz}$ range for the present measurements, and $\sim 7 \mathrm{MHz}$ for the IR measurements, resulting in a weighted standard deviation of the fit $\sigma_{w}=1.02$. The accuracy of the spectroscopic parameters of this work is marginally improved (a factor of $\sim 2-3$ ) with respect to the former work, since only five rotational lines could be recorded in high resolution but eight parameters are fit. It may be also noted that the $\delta_{k}$ parameter is not significantly different from zero in this fit.
For $\mathrm{NHD}_{3}^{+}$, we only have the three presently measured transition frequencies available; therefore, we cannot provide a least squares fit. Furthermore, since the transitions obey $\Delta K=0$, we cannot determine $C$ and $D_{K}$. With the three experimental frequencies, we have determined $B, D_{J}$, and $D_{J K}$, as shown in Table 2. Also listed in Table 2 are the values quoted by Chang \& Nesbitt (2013). Our quoted errors are obtained by propagating the experimental uncertainties through the closed linear relations existing between the three observed frequencies and the three retrieved constants. We assumed that there is no correlation between the experimental frequencies, thus the uncertainties are just added in quadrature with the appropriate coefficients.

\section{Conclusion and Outlook}

We measured, with $\mathrm{kHz}-\mathrm{level}$ accuracies, rotational transitions originating from the lowest energy levels of the deuterated ammonium isotopologues $\mathrm{NH}_{3} \mathrm{D}^{+}, \mathrm{NH}_{2} \mathrm{D}_{2}^{+}$, and $\mathrm{NHD}_{3}^{+}$. Prior to this work, $\mathrm{NH}_{3} \mathrm{D}^{+}$was already identified in Orion IRc2 and Barnard B1b (Cernicharo et al. 2013). For the $\mathrm{NH}_{2} \mathrm{D}_{2}^{+}$and $\mathrm{NHD}_{3}^{+}$isotopologues, these rest frequencies will facilitate the search for these ions in the ISM. Cold dark clouds that are highly depleted in $\mathrm{C}$ and $\mathrm{O}$ are the kind of sources where high-deuterium fractionation is expected. The cold protostellar core B1b is a source where the abundance of deuterated isotopologues of ammonia and the diazenylium ion $\left(\mathrm{N}_{2} \mathrm{H}^{+}\right)$is quite remarkable, as is the presence of other multiply deuterated species, like $\mathrm{D}_{2} \mathrm{CS}$ (Marcelino et al. 2005) or $\mathrm{D}_{2} \mathrm{~S}$ (Vastel et al. 2003). The chemical model used in the interpretation of $\mathrm{NH}_{3} \mathrm{D}^{+}$observations in $\mathrm{B} 1 \mathrm{~b}$ predicted that the abundance of $\mathrm{NH}_{2} \mathrm{D}_{2}^{+}$could be just a factor of two lower 
than that of $\mathrm{NH}_{3} \mathrm{D}^{+}$. Therefore, one could expect the detection of more deuterated species of the ammonium ion in this or other cold dark clouds. We note, however, that the partition function of $\mathrm{NH}_{2} \mathrm{D}_{2}^{+}$, being an asymmetric top, is more unfavorable, and, even at the low temperature of $\mathrm{B} 1 \mathrm{~b}$ ( $T \sim 12 \mathrm{~K}$ ), spectral dilution is to be expected. The slightly higher electric-dipole moment $(0.29 \mathrm{D}$ versus $0.26 \mathrm{D})$ just marginally improves this situation. Although the necessary sensitivity for this detection seems demanding, the ever increasing capabilities of receivers and observatories will likely make it possible in the not-so-distant future. $\mathrm{NHD}_{3}^{+}$has a smaller abundance and dipole moment, but it has higher symmetry (it is a symmetric top, like $\mathrm{NH}_{3} \mathrm{D}^{+}$), and, therefore, a more favorable partition function, so it may also be soon within the reach of observational radio astronomy. In any case, the frequencies provided in this work will enable those detections.

This work (including a research stay of J.L.D. in Köln) has been supported by the Deutsche Forschungsgemeinschaft (DFG) via SFB 956 project B2 and the Gerätezentrum "Cologne Center for Terahertz Spectroscopy." J.L.D. acknowledges partial financial support from the Spanish MINECO through grant FIS2016-77726-C3-1-P and from the European Research Council through grant agreement ERC-2013-SyG610256-NANOCOSMOS. The authors gratefully acknowledge the work done over the last years by the electrical and mechanical workshops of the I. Physikalisches Institut. We thank Marius Hermanns, Frank Lewen, and Matthias Töpfer for assistance in the measurements.

Software: PGOPHER (Western 2017).

Note Added in Proof. At the proof stage of this paper, we learned of the very recent publication of the infrared spectrum of the $\mathrm{NHD}_{3}^{+} \nu_{1}$ band (Chang et al. 2018). A combined analysis of the present rotational data together with the new available combination differences has been performed, allowing us to do a least squares fit. The new values thus derived for the ground state rotational constants of $\mathrm{NHD}_{3}^{+}$are (in $\mathrm{MHz}$ ) $B=111117.97939(48), D_{J}=1.75390(15)$, and $D_{J K}=-1.37627(95)$.

\section{ORCID iDs}

José L. Doménech @i https://orcid.org/0000-0001-8629-2566

Stephan Schlemmer (1) https://orcid.org/0000-0002-1421-7281

Oskar Asvany (i) https://orcid.org/0000-0003-2995-0803

\section{References}

Aikawa, Y., Wakelam, V., Garrod, R. T., \& Herbst, E. 2008, ApJ, 674, 984 Asvany, O., Bielau, F., Moratschke, D., Krause, J., \& Schlemmer, S. 2010, RScI, 81, 076102

Asvany, O., Brünken, S., Kluge, L., \& Schlemmer, S. 2014, ApPhB, 114, 203

Brünken, S., Kluge, L., Stoffels, A., Asvany, O., \& Schlemmer, S. 2014, ApJL, 783, L4

Brünken, S., Kluge, L., Stoffels, A., Pérez-Ríos, J., \& Schlemmer, S. 2017, JMoSp, 332, 67

Cernicharo, J., Tercero, B., Fuente, A., et al. 2013, ApJ, 771, L10

Chang, C.-H., \& Nesbitt, D. J. 2013, in 68th OSU Int. Symp. on Molecular Spectroscopy, Contribution MG03, https://kb.osu.edu/handle/1811/55596

Chang, C.-H., \& Nesbitt, D. J. 2018, JChPh, 148, 014304

Chang, C.-H., Scrape, P. G., \& Nesbitt, D. J. 2018, JChPh, 149, 144303

Cheung, A. C., Rank, D. M., Townes, C. H., Thornton, D. D., \& Welch, W. J. 1968, PhRvL, 21, 1701

Doménech, J. L., Cueto, M., Herrero, V. J., et al. 2013, ApJL, 771, L11

Doménech, J. L., Jusko, P., Schlemmer, S., \& Asvany, O. 2018, ApJ, 857, 61

Doménech, J. L., Schlemmer, S., \& Asvany, O. 2017, ApJ, 849, 60

Gerin, M., Marcelino, N., Biver, N., et al. 2009, A\&A, 498, L9

Grozdanov, T. P., McCarroll, R., \& Roueff, E. 2016, A\&A, 589, A105

Harada, N., Herbst, E., \& Wakelam, V. 2010, ApJ, 721, 1570

Harju, J., Daniel, F., Sipilä, O., et al. 2017, A\&A, 600, A61

Hugo, E., Asvany, O., \& Schlemmer, S. 2009, JChPh, 130, 164302

Jusko, P., Stoffels, A., Thorwirth, S., et al. 2017, JMoSp, 332, 59

Knauth, D. C., Andersson, B.-G., McCandliss, S. R., \& Warren Moos, H. 2004, Natur, 429, 636

Linsky, J. L., Draine, B. T., Moos, H. W., et al. 2006, ApJ, 647, 1106

Marcelino, N., Cernicharo, J., Roueff, E., Gerin, M., \& Mauersberger, R. 2005, ApJ, 620, 308

Millar, T. J. 2003, SSRv, 106, 73

Nakanaga, T., \& Amano, T. 1986, CaJPh, 64, 1356

Nejad, L. A. M., Williams, D. A., \& Charnley, S. B. 1990, MNRAS, 246, 183

Stoffels, A., Kluge, L., Schlemmer, S., \& Brünken, S. 2016, A\&A, 593, A56

Vastel, C., Phillips, T. G., Ceccarelli, C., \& Pearson, J. 2003, ApJ, 593, L97

Western, C. M. 2017, JQSRT, 186, 221

Zymak, I., Hejduk, M., Mulin, D., et al. 2013, ApJ, 768, 86 